\title{
SUSY flavour / Flavour sector in BSM scenarios
}

\author{
Andreas Crivellin ${ }^{* \dagger}$ \\ Paul Scherrer Institut, CH-5232 Villigen PSI, Switzerland \\ E-mail: andreas.crivellinepsi.ch
}

In these proceedings I review the status of flavour physics beyond the Standard Model. While the LHC did not directly observe any new particles yet, LHCb, BELLE and BABAR found intriguing hints for the violation of lepton flavour universality and also the measurement of the anomalous magnetic moment of the muon can be interpreted as a sign of such a violation. In addition, recent lattice results point towards new sources of $\mathrm{CP}$ violation in $\varepsilon^{\prime} / \varepsilon$. I discuss how these hints for new physics can be explained within various extensions of the Standard Model, including supersymmetry. It turns out that leptoquarqs and $Z^{\prime}$ bosons are prime candidates for explaining these anomalies while the MSSM can only account for the anomalous magnetic moment of the muon and $\varepsilon^{\prime} / \varepsilon$. However, one should keep in mind that any model can be supersymmetrized.

The 15th International Conference on Flavor Physics \& CP Violation

5-9 June 2017

Prague, Czech Republic

\footnotetext{
* Speaker.

${ }^{\dagger}$ I thank the organizers for the invitation and the opportunity to present my research. This work is supported by an Ambizione Grant of the Swiss National Science Foundation (PZ00P2_154834).
} 


\section{Introduction}

While the LHC did not directly observe any particle beyond the ones of the Standard Model (SM), including SUSY particles, in the recent years intriguing hints for the violation of lepton flavour universality (LFU) have been accumulated. In the semi-leptonic $B$ decays

$$
\begin{aligned}
& R\left(D^{(*)}\right)=\left(B \rightarrow D^{(*)} \tau v\right) /\left(B \rightarrow D^{(*)} \ell v\right), \\
& R(J / \Psi)=\left(B_{c} \rightarrow J / \Psi \tau v\right) /\left(B_{c} \rightarrow J / \Psi \ell v\right), \\
& R\left(K^{(*)}\right)=\left(B \rightarrow K^{(*)} \mu^{+} \mu^{-}\right) /\left(B \rightarrow K^{(*)} e^{+} e^{-}\right),
\end{aligned}
$$

sizable deviations from the SM predictions have been observed. In addition, there is the longstanding discrepancy in the anomalous magnetic moment of the muon $\left(a_{\mu}\right)$ which can also be interpreted as a sign of lepton flavour universality (LFU) violation.

Let us consider the current situation in some more detail: in $R\left(D^{(*)}\right)$ BaBar [1], BELLE [2,3] and $\mathrm{LHCb}[4,5]$ found deviations from the SM predictions with a combined statistical significance for LFUV of $\approx 4 \sigma[6]$. Very recently, LHCb measured $R(J / \Psi)$ finding a value which exceeds the SM prediction by approximately $2 \sigma$ [7] which is in agreement with the expectations from the $R\left(D^{(*)}\right)$ measurements and further strengthens the significance for NP in $b \rightarrow c \tau v$ transitions.

Concerning $b \rightarrow s \ell^{+} \ell^{-}$processes, $R(K)$ [8] and $R\left(K^{*}\right)$ [9] show a combined significance for LFU violation at the $4 \sigma$ level [10-14]. Taking into account all other $b \rightarrow s \mu^{+} \mu^{-}$observables (like $\left.P 5^{\prime}[15]\right)$ as well, the global fit even shows compelling evidence for NP above the $5 \sigma$ level [16] for several scenarios like NP in $C_{9}$ only, $C_{9}=-C_{10}$ or $C_{9}=-C_{9}^{\prime}$.

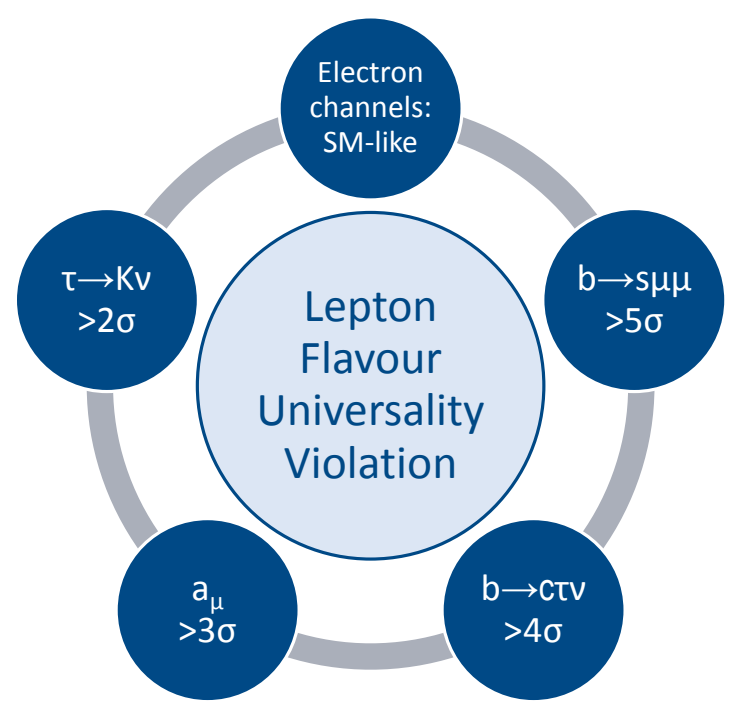

Figure 1: Hints for LFU violating NP.

Furthermore, there is the long-standing discrepancy in $a_{\mu}$, i.e. the tension between the measurement [17] and the SM prediction is at the $3 \sigma$ level [18]. Anomalous magnetic moments are already in the SM flavour non-universal. Nevertheless, if NP coupled with the same strength to electrons and to muons, $a_{e}$ would be more sensitive. Therefore, since no deviation from the SM 
in $a_{e}$ has been observed, the tension in $a_{\mu}$ can be considered as another sign for LFU violating NP. In addition $V_{u s}$ extracted from tau lepton decays shows a tension of $2.5 \sigma$ with the value of $V_{u s}$ determined from CKM unitarity [19]. Since in $\tau \rightarrow K v$ a heavy tau lepton is involved while in kaon decays like $K \rightarrow \ell v$ or $K \rightarrow \pi \ell v$ only light leptons are involved, this can be interpreted as a sign of LFU violation as well.

All these measurements together form a coherent picture and strongly point towards LFU violating NP as shown in Fig. 1.

In addition to these hints for LFU violation, there are tensions between the theory predictions for $\varepsilon^{\prime} / \varepsilon$ (measuring CP violation in Kaon decays) of almost $3 \sigma$ [20] and for the measured CP asymmetry in $\tau \rightarrow K_{s} \pi v$ at $2.8 \sigma$ [21]. Even though, from a first point of view, these anomalies are unrelated to the ones pointing at LFU violation mentioned above, a UV complete model might still provide a correlation among them.

We will discuss each of these anomalies in the next sections. As we will see, most of the anomalies can be explained using leptoquarks (LQ). Therefore, we will study combined solutions using these particles in Sec. 6.

\section{2. $b \rightarrow c \tau v$}

The observables $R\left(D^{(*)}\right)$ and $R(J / \Psi)$ involving $b \rightarrow c \tau v$ transitions are charged current processes mediated in the SM at tree-level by a $W$ boson. Since the relative effect of NP must be of the order of $10 \%$ to account for the measured values, also NP needs to contribute at tree-level. Therefore, a solution via charged Higgses (see for example [22-26]), by LQs [27-31] or a $W^{\prime}$ boson [32] is in principle possible. However, recent bounds from the $B_{c}$ lifetime [33], $q^{2}$ distributions [34] and direct LHC searches [35] rule out, or at least strongly disfavour Higgses and $W^{\prime}$ bosons. Therefore, we are left with LQs which, despite the bounds from EW precision data [36], still provide a valid explanation [37-40].

Concerning SUSY, the leptoquark $S U(2)$ singlet $S_{1}$, which is embedded in the R-Parity violating MSSM as the right-handed bottom squark, can in principle explain the $b \rightarrow c \tau \nu$ data. However, due to the bounds from $b \rightarrow s v v$ it cannot account fully for the experimental data [41,42] even though the effect goes in the right direction.

\section{3. $b \rightarrow s \ell^{+} \ell^{-}$}

$b \rightarrow s \ell^{+} \ell^{-}$, being a flavour changing neutral current, is in the SM suppressed both by a loop and a CKM factor. Therefore, the NP effect does also not have to be very large to account for the measured data which deviate from the SM prediction by around 20\%. This tension can be explained by a heavy neutral gauge boson $Z^{\prime}$ (e.g. [43-50]) or, again by LQs (see e.g. [51,52]). Alternative solutions include box contributions of heavy new scalars and fermions [53,54] or top-induced loop effects $[55,56]$.

Here a full solution within the MSSM is not possible [57], i.e. one cannot account for the central value. However, one can still improve the fit compared to the SM [58]. 

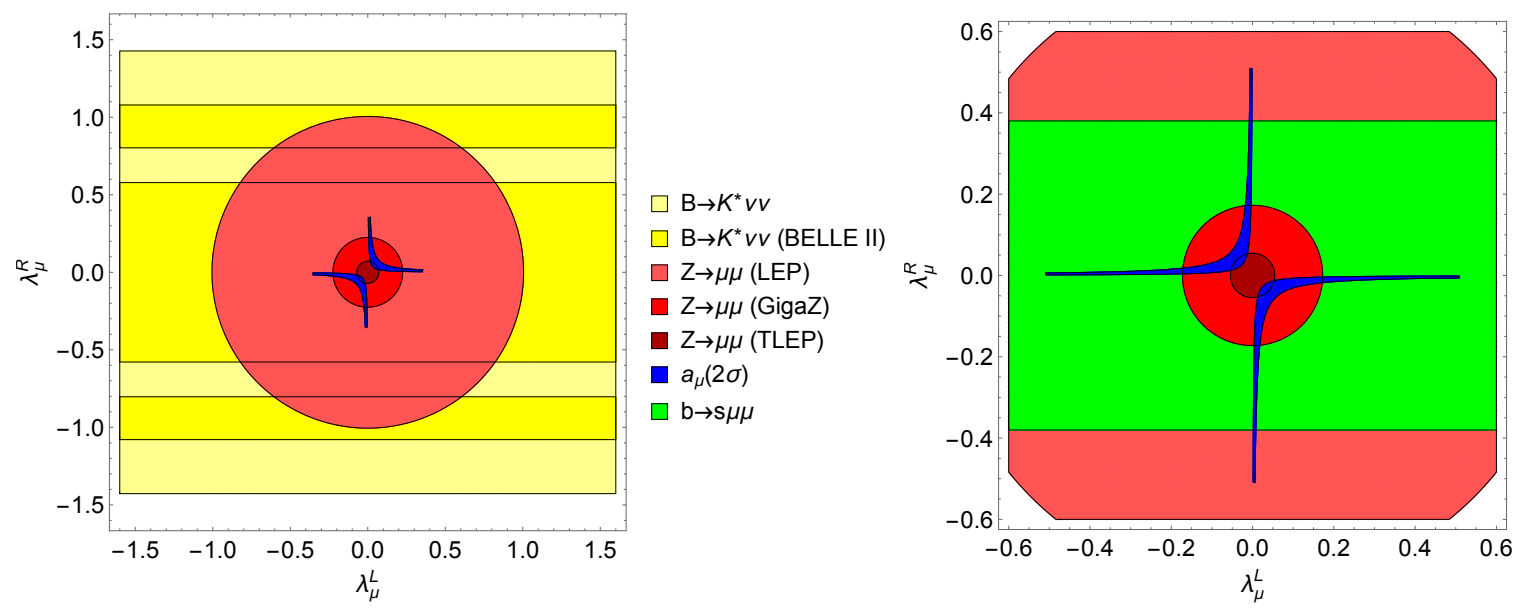

Figure 2: Left: Allowed regions in the $\lambda_{\mu}^{L}-\lambda_{\mu}^{R}$ plane from current and future experiments for $S U(2)$ singlet leptoquarks $\Phi_{1}$ with $M=1 \mathrm{TeV}$. Right: Same as the left plot for the $S U(2)$ doublet leptoquark $\Phi_{2}$.

4. $a_{\mu}$

For the anomaly in $a_{\mu}$, many kinds of new particles can give the desired effect. In fact, it is well known that the MSSM can account for the measurement by $\tan \beta$ enhanced slepton contributions (see for example Ref. [59] for an overview) or via a large $A_{22}^{\ell}$ term [60].

An alternative explanation of the AMM of the muon involves leptoquarks [61]. Here, even though the leptoquark must be rather heavy due to LHC constraints, one can still get sizable effects in the AMM since the amplitude can be enhanced by $m_{t} / m_{\mu}$ compared to the SM. In fact, among the 5 scalar leptoquark representations which are invariant under the SM gauge group [62], only two can in principle generate these enhanced effects as they possess couplings to left- and righthanded muons simultaneously:

- $\Phi_{1}: S U(2)_{L}$ singlet with hypercharge $-2 / 3$.

- $\Phi_{2}: S U(2)_{L}$ doublet with hypercharge $-7 / 3$.

In addition to the desired effect in the AMM of the muon also $Z \rightarrow \mu^{+} \mu^{-}$and $b \rightarrow s \mu^{+} \mu^{-}$(for $\Phi_{2}$ ) or $b \rightarrow s v \bar{v}$ (for $\Phi_{1}$ ) are affected (see Ref. [63] for details). The allowed regions from these processes are shown in Fig. 2 for a leptoquark mass of $1 \mathrm{TeV}$. This is save with respect to direct searches. For $Z \rightarrow \mu^{+} \mu^{-}$also the expected future bounds at GigaZ are shown where one can expect an increase of precision by a factor of around 20 [64]. Also the projected TLEP bounds and BELLE II limits for $B \rightarrow K^{(*)} v \bar{v}$ are depicted.

5. $\varepsilon^{\prime} / \varepsilon$

For $\varepsilon^{\prime} / \varepsilon$ the most natural solution is probably a $Z^{\prime}$ boson $[65,66]$ but also models with modified $Z$ couplings $[65,67]$ or a modified $W$ coupling [68] work.

Here, also the plain MSSM could explain the deviations [69-71]. The desired effect in $\varepsilon^{\prime}$ can be generated via gluino-squark boxes [69] together with a simultaneous efficient suppression 
of the supersymmetric QCD contributions to $\varepsilon_{K}$ [72]. In this case interesting correlations with $\mathscr{B}\left(K_{L} \rightarrow \pi^{0} v \bar{v}\right)$ and $\mathscr{B}\left(K^{+} \rightarrow \pi^{+} v \bar{v}\right)$ occur which are shown in Fig. 3.
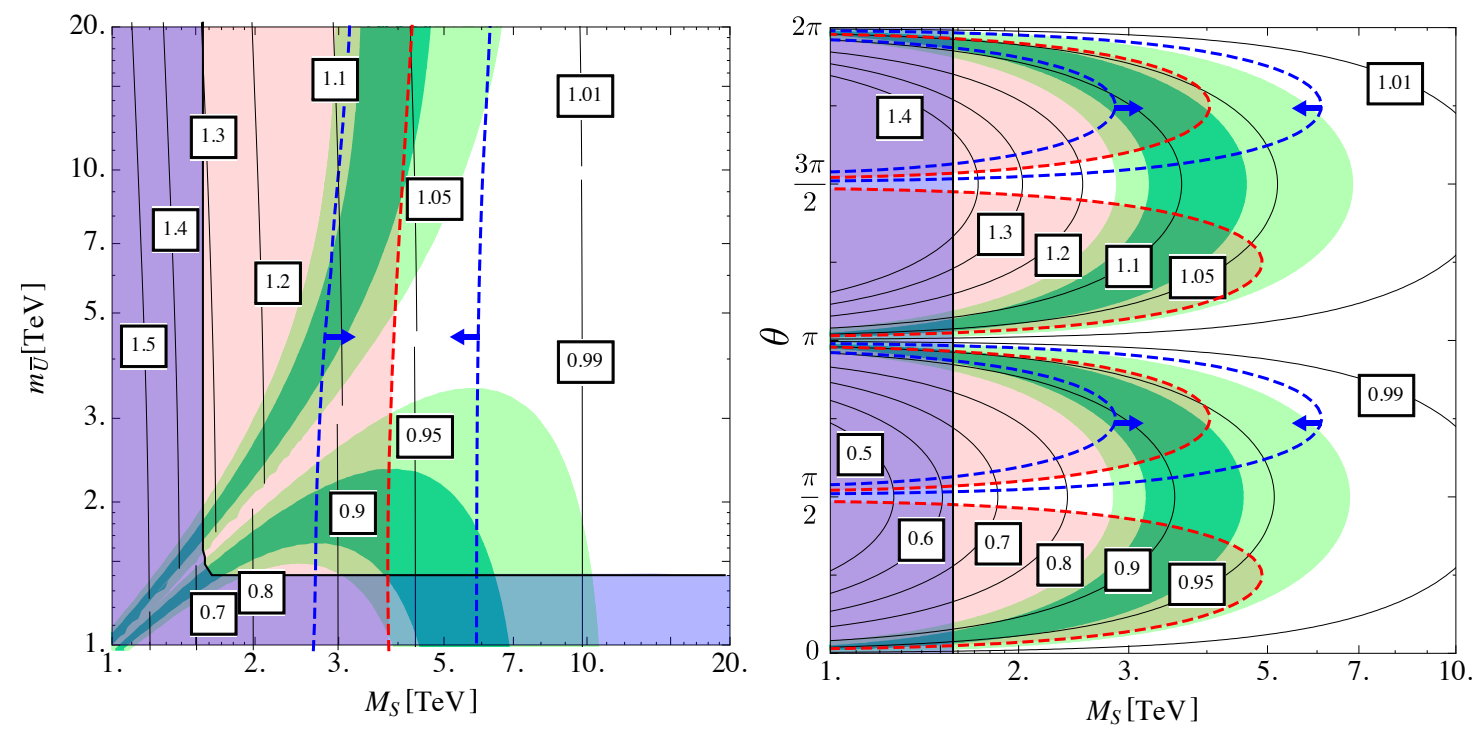

Figure 3: Contours of $\mathscr{B}\left(K_{L} \rightarrow \pi^{0} v \bar{v}\right) / \mathscr{B}^{\mathrm{SM}}\left(K_{L} \rightarrow \pi^{0} v \bar{v}\right)$. The $\varepsilon_{K}^{\prime} / \varepsilon_{K}$ discrepancy is resolved at the $1 \sigma(2 \sigma)$ level within the dark (light) green region. The red shaded region is excluded by $\varepsilon_{K}$ at $95 \%$ C.L. using the inclusive value $\left|V_{c b}\right|$, while the region between the blue-dashed lines can explain the $\varepsilon_{K}$ discrepancy which is present if the exclusive determination of $V_{c b}$ is used [73]. The blue shaded region is excluded by the current LHC results from CMS and ATLAS [74-76]. $M_{3} / M_{S}=1.5, m_{L}=300 \mathrm{GeV}$ and GUT relations among gaugino masses are used. In the left plot, $\Delta_{Q, 12}=0.1 \exp (-i \pi / 4)$ for $m_{\bar{U}}>m_{\bar{D}}=m_{Q}=M_{S}$ (upper branch) and $\Delta_{Q, 12}=0.1 \exp (i 3 \pi / 4)$ for $m_{\bar{U}}<m_{\bar{D}}=m_{Q}=M_{S}$ (lower branch). In the right plot, $\left|\Delta_{Q, 12}\right|=0.1$ is used, $m_{\bar{D}}=2 m_{\bar{U}}=2 m_{Q}=2 M_{S}$ (for $\left.0<\theta<\pi\right)$ and $m_{\bar{U}}=2 m_{\bar{D}}=2 m_{Q}=2 M_{S}($ for $\pi<\theta<2 \pi)$.

\section{Simultaneous explanations with Leptoquarks}

Among the 10 representations of leptoquarks which couple to SM fermions there are 5 vector LQs and 5 scalar LQs.

\subsection{Scalar leptoquarks}

As discussed above the scalar LQ $S U(2)$ singlet cannot fully account for the $b \rightarrow c \tau v$ anomalies. Furthermore, its loop-effects in $b \rightarrow s \mu^{+} \mu^{-}$are also too small due to the constraints from $B \rightarrow D \mu v / B \rightarrow D e v$. Therefore, a second LQ is required to explain both anomalies, the $S U(2)$ triplet $\Phi_{3}$. These LQs couple to fermions in the following way:

$$
L=\lambda_{f i}^{1 L} \overline{Q_{f}^{c}} i \tau_{2} L_{i} \Phi_{1}^{\dagger}+\lambda_{f i}^{3 L} \overline{Q_{f}^{c}} i \tau_{2}\left(\tau \cdot \Phi_{3}\right)^{\dagger} L_{i}+\text { h.c. } .
$$

As shown in Fig. 4 we see that a cancellation at the $10 \%$ level between the two contributions is required. Therefore, we will in the following assume that both leptoquarks have the same mass $M$ and that their effect in $b \rightarrow s v \bar{v}$ cancel we impose the discrete symmetry

$$
\lambda_{j k}^{L} \equiv \lambda_{j k}^{1 L}, \quad \lambda_{j k}^{3 L}=e^{i \pi j} \lambda_{j k}^{L}
$$


on the couplings to fermions.

Let us first consider the size of the couplings needed to explain $R(D)$ and $R\left(D^{*}\right)$. Here and in the following, we will assume them to be real. We only need small couplings (of the order of 0.1 for $1 \mathrm{TeV}$ leptoquarks) in order to explain $R(D), R\left(D^{*}\right)$. This is possible because we avoid contributions to $b \rightarrow s v \bar{v}$ and hence, our effect in $b \rightarrow c \tau v$ does not need to be CKM suppressed. Therefore, the bounds from Ref. [35] do not apply to our model and we are not in conflict with LHC bounds, especially because the LQs can be much heavier than $1 \mathrm{TeV}$ while still possessing perturbative couplings and explaining $R\left(D^{(*)}\right)$.

Next, note that neglecting small CKM factors, the contributions to $b \rightarrow c \tau v$ and $b \rightarrow s \tau^{+} \tau^{-}$ depend on the same product of couplings $\lambda_{23}^{L} \lambda_{33}^{L *}$ (modulus small CKM ratios). Therefore, we can express $B_{s} \rightarrow \tau^{+} \tau^{-}$in terms of the effect in $R\left(D^{(*)}\right)$ :

$$
\frac{\operatorname{Br}\left(B_{s} \rightarrow \tau \tau\right)}{\operatorname{Br}\left(B_{S} \rightarrow \tau \tau\right)_{\mathrm{SM}}}=\left(1+2 \frac{\pi}{\alpha} \frac{V_{c b}}{V_{t s}^{*}} \frac{\sqrt{X_{D^{(*)}}}-1}{C_{10}^{\mathrm{SM}}}\right)^{2} .
$$

The $B_{s} \rightarrow \tau^{+} \tau^{-}$branching ratio can be enhanced by up to three orders of magnitude compared to the SM prediction. Therefore, even though it is experimentally challenging to search for, our model can be tested with $B_{s} \rightarrow \tau^{+} \tau^{-}$measurements at LHCb. Also an enhancement of $B \rightarrow K^{(*)} \tau^{+} \tau^{-}$in the same ballpark is predicted which could be tested at BELLE II.

Let us now consider the effect of including $b \rightarrow s \mu^{+} \mu^{-}$transitions in our analysis. In this case effects in $B \rightarrow D^{(*)} \mu v / B \rightarrow D^{(*)} e v$ are predicted if still addressing $R(D)$ and $R\left(D^{*}\right)$ simultaneously. We checked that the effect is at the per-mill level which is compatible with BELLE and BABAR measurements ${ }^{1}$. However, interesting correlations with $b \rightarrow s \tau \mu$ processes appear. Here we find

$$
C_{9}^{32}=-2 \frac{\pi}{\alpha} \frac{V_{c b}}{V_{t s}^{*}} \frac{\lambda_{32}^{L}}{\lambda_{33}^{L}}\left(\sqrt{X_{D^{(*)}}}-1\right), \quad C_{9}^{23}=\frac{\lambda_{33}^{L}}{\lambda_{32}^{L}} C_{9}^{22},
$$

which depends only on the ratio $\lambda_{33}^{L} / \lambda_{32}^{L}$ as a free parameter. Note that the dependence on $C_{9}^{22}$ is much weaker than on $X_{D^{(*)}}$. The resulting bounds and predictions are shown in the left plot of Fig. 5. We take the experimental limit [79]

$$
\mathrm{Br}[B \rightarrow K \tau \mu]<4.8 \times 10^{-5} .
$$

Note that $R\left(D^{(*)}\right)$ can only be fully explained for $\lambda_{33}^{L} / \lambda_{32}^{L}>1$.

Considering only the couplings $\lambda^{L}$ the effect in $\tau \rightarrow \mu \gamma$ is negligibly small. Things get much more interesting if we aim at a simultaneous explanation of the anomalous magnetic moment of the muon. In this case chirally enhanced effects also appear in $\tau \rightarrow \mu \gamma$. We have

$$
\operatorname{Br}[\tau \rightarrow \mu \gamma] \geq \frac{\alpha m_{\tau}^{3}}{16 \Gamma_{\tau}} \frac{a_{\mu}^{2}}{m_{\mu}^{2}}\left|\frac{\lambda_{33}^{L}}{\lambda_{32}^{L}}\right|^{2}
$$

Here we set $\lambda_{33}^{R}=0$.

\footnotetext{
${ }^{1}$ This is contrary to Ref. [29] which cannot explain $R\left(D^{(*)}\right)$ and $b \rightarrow s \mu^{+} \mu^{-}$data simultaneously without violating the bounds from $B \rightarrow D^{(*)} \mu v / B \rightarrow D^{(*)} e v$ as pointed out in Ref. [77]. However, this tension can be relieved with leptoquark masses larger than $5 \mathrm{TeV}$ [78].
} 


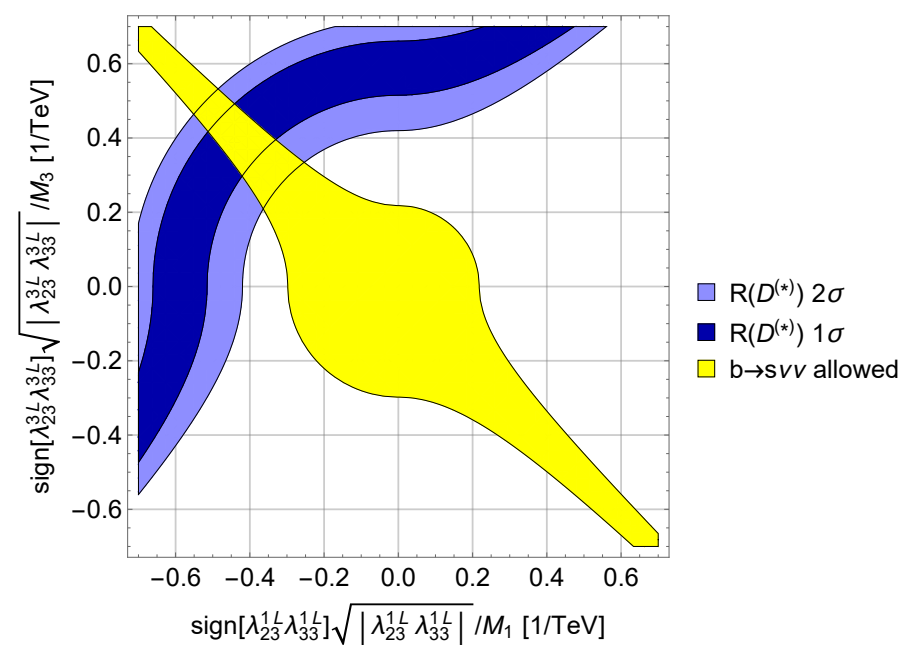

Figure 4: Allowed regions for $R\left(D^{(*)}\right)$ and $b \rightarrow s v v$ assuming independent couplings and masses for the leptquark singlet and triplet. Here $M_{1(3)}$ is the mass of $\Phi_{1}\left(\Phi_{3}\right)$.

Note that $\operatorname{Br}(\tau \rightarrow \mu \gamma)$ can only be enhanced by allowing $\lambda_{33}^{R}$ to be different from zero, resulting in the $\geq$ sign in eq. (6.6). The result is shown in the right plot of Fig. 5. Note that $a_{\mu}$ can only be explained for $\lambda_{33}^{L} / \lambda_{32}^{L}<0.65$ (at the $2 \sigma$ level). This is opposite to the case of $b \rightarrow s \mu^{+} \mu^{-}$ which can only be explained for $\lambda_{33}^{L} / \lambda_{32}^{L}>1$. Therefore, we conclude that our model can explain out of the three anomalies $R\left(D^{(*)}\right), b \rightarrow s \mu^{+} \mu^{-}$and $a_{\mu}$ only two simultaneously.

\subsection{Vector leptoquarks}

The vector leptoquark $S U(2)$ singlet with hypercharge $-4 / 3$ is a natural candidate for a simultaneous explanation of $R(D)$ and $R\left(D^{*}\right)$ together with $b \rightarrow s \ell^{+} \ell^{-}$data since it avoids the stringent bounds from $B \rightarrow K^{(*)} v v$. Interestingly, this vector leptoquark is contained within the theoretically very appealing PS model as a $S U(4)$ gauge boson. In the conventional model, the bounds on the symmetry breaking scale from $K_{L} \rightarrow \mu e$ and $K \rightarrow \pi \mu e$ are so strong (at the PeV scale) [80] that any other observable effects in flavour physics are ruled out from the outset. However, in Ref. [40] a model with additional vector-like fermions was constructed which can avoid these bounds.

In order to get a sizable effect, the mixing of the vector like lepton with the tau lepton $\left(\ell_{3}^{L}\right)$ should be large. Assuming it to be maximal, we show the regions preferred by $R\left(D^{(*)}\right)$ in the left plot of Fig. 6 for $M_{L Q}=1.5 \mathrm{TeV}$. From this we can see that also the mixing between $Q_{3}^{L}$ and $q_{3}^{L}$ $\left(s_{3}^{Q}\right)$, as well as the misalignment between the quark and lepton Yukawa couplings of the second and third generations $\left(s_{23}^{q \ell}\right)$ should be sizable. Our model predicts a significant enhancement of $B_{s} \rightarrow \tau^{+} \tau^{-}$compared to the SM prediction.

Let us now turn to the explanation of $b \rightarrow s \ell^{+} \ell^{-}$data. Assuming the absence of mixing with leptons of the first generation, we are safe from processes like $\mu \rightarrow e \gamma$ or $b \rightarrow s \mu e$ [81] and get the right effect in $R(K)$ and $R\left(K^{*}\right)$. Assuming maximal mixing for the third generation quarks and leptons, we show the preferred region from $b \rightarrow s \ell^{+} \ell^{-}$in the right plot of Fig. 6. This region overlaps with the one from $R\left(D^{(*)}\right)$ for small mixing between the second generation fermions $\left(s_{2}^{Q, L}\right)$ where the predicted branching ratio for $B \rightarrow K \tau \mu$ is automatically compatible with 

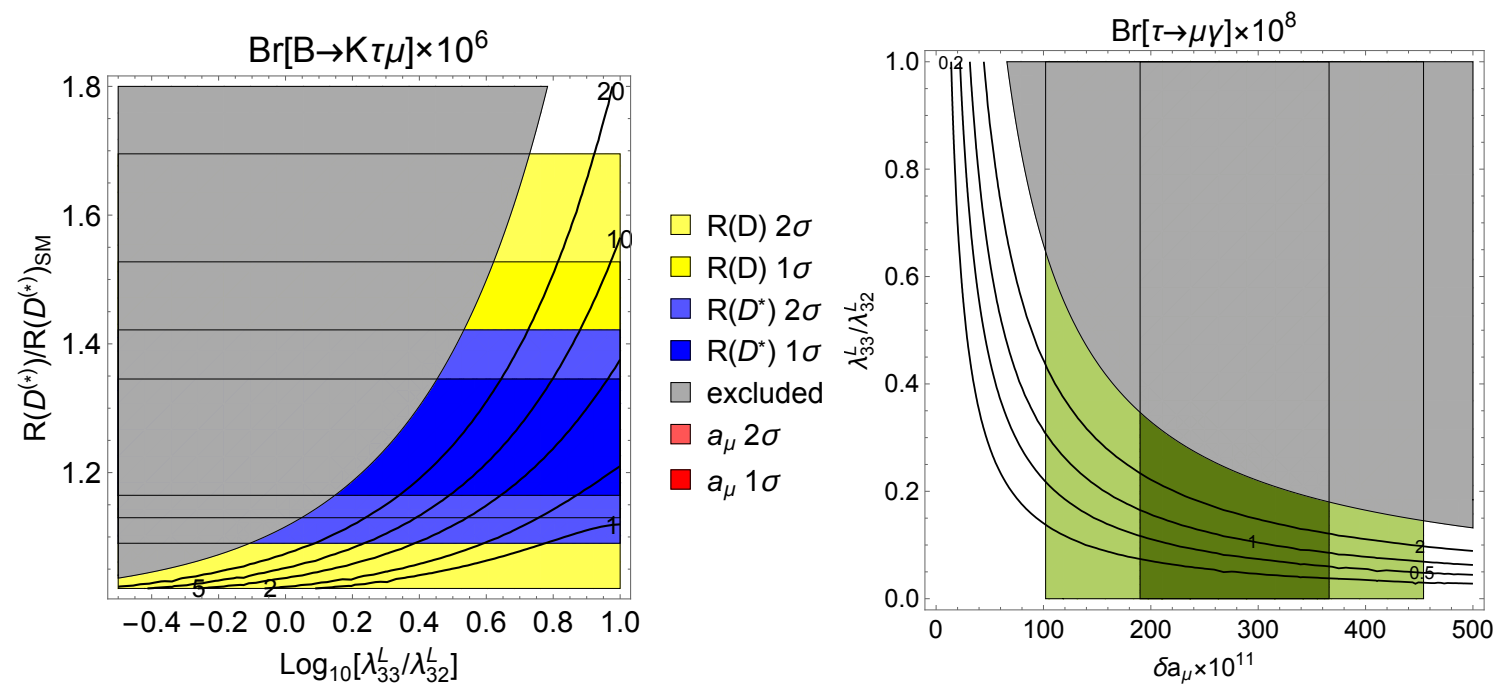

Figure 5: Left: Contours and excluded region for $B \rightarrow K \tau \mu=\left(B \rightarrow K \tau^{+} \mu^{-}+B \rightarrow K \tau^{-} \mu^{+}\right) / 2$ for $C_{9}^{22}=-0.5$, i.e. assuming that $C_{9}^{22}$ takes the central value obtained from the $b \rightarrow s \mu^{+} \mu^{-}$fit. The coloured regions are allowed by the various processes. For $R(D)$ and $R\left(D^{*}\right)$ we used again the weighted average for $R\left(D^{(*)}\right)_{\mathrm{EXP}} / R\left(D^{(*)}\right)_{\mathrm{SM}}$. Right: The contour lines show $\operatorname{Br}[\tau \rightarrow \mu \gamma] \times 10^{8}$. The gray region is excluded by

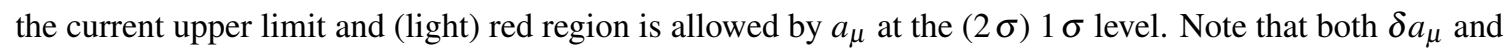
$\tau \rightarrow \mu \gamma$ are only a function of $\lambda_{33}^{L} / \lambda_{32}^{L}$ and therefore independent of $b \rightarrow s \mu^{+} \mu^{-}$transitions.

the experimental bounds. However, the predicted rate is still sizable and well within the reach of future measurements.

\section{Conclusions}

In these proceedings we reviewed the currents hints for lepton flavour universality violation in $b \rightarrow s \mu^{+} \mu^{-}$and $b \rightarrow c \tau \nu$ processes as well as $\varepsilon^{\prime} / \varepsilon$ and the anomalous magnetic moment of the muon. For $b \rightarrow s \mu^{+} \mu^{-}, b \rightarrow c \tau \nu$ and $a_{\mu}$ leptoquarks provide a natural explanation. Alternatively, $b \rightarrow s \mu^{+} \mu^{-}$data and $\varepsilon^{\prime} / \varepsilon$ can be explained by a $Z^{\prime}$ boson. While the plain MSSM can only account for the anomaly in $\varepsilon^{\prime} / \varepsilon$, one should keep in mind that any model can be made supersymmetric by promoting fields to supermultiplets.

\section{References}

[1] J. P. Lees et al. [BaBar Collaboration], Phys. Rev. Lett. 109, 101802 (2012) doi:10.1103/PhysRevLett.109.101802 [arXiv:1205.5442 [hep-ex]].

[2] M. Huschle et al. [Belle Collaboration], Phys. Rev. D 92, no. 7, 072014 (2015) doi:10.1103/PhysRevD.92.072014 [arXiv:1507.03233 [hep-ex]].

[3] S. Hirose et al. [Belle Collaboration], arXiv:1709.00129 [hep-ex].

[4] R. Aaij et al. [LHCb Collaboration], Phys. Rev. Lett. 115, no. 11, 111803 (2015) Erratum: [Phys. Rev. Lett. 115, no. 15, 159901 (2015)] doi:10.1103/PhysRevLett.115.159901, 10.1103/PhysRevLett.115.111803 [arXiv:1506.08614 [hep-ex]]. 

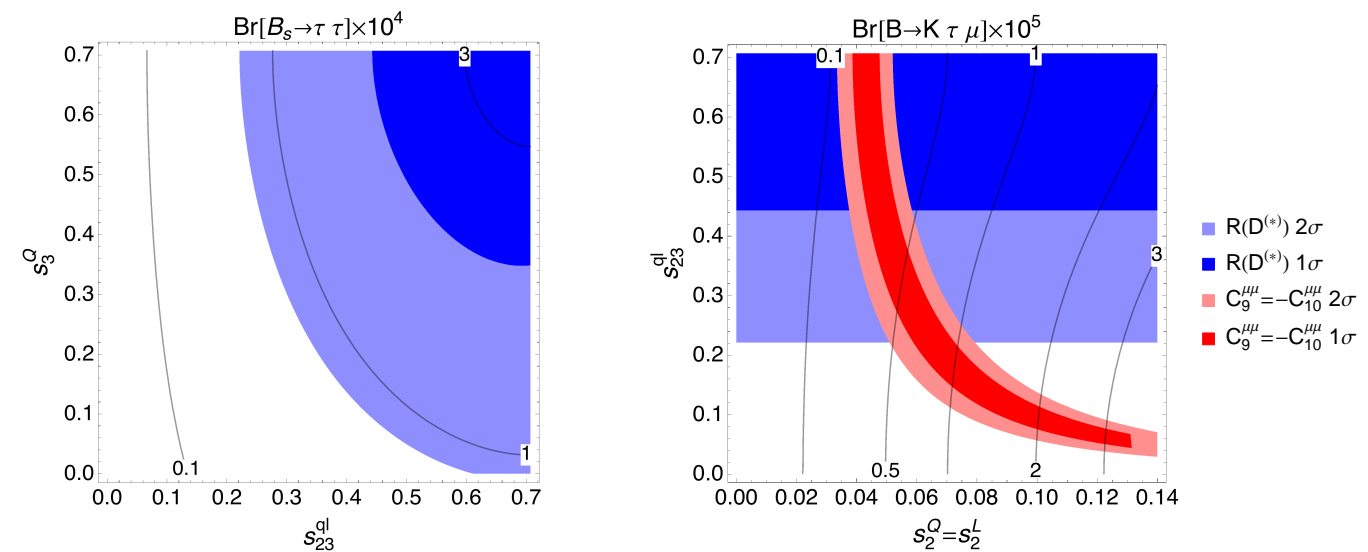

Figure 6: Left: Allowed regions from $R\left(D^{(*)}\right)$ for $M_{L Q}=1.5 \mathrm{TeV}$ and $s_{3}^{Q}=s_{3}^{L}=1 / \sqrt{2}$. Here we used the weighted average for $R(D)$ and $R\left(D^{*}\right)$. The contour lines denote $\operatorname{Br}\left(B_{s} \rightarrow \tau^{+} \tau^{-}\right) \times 10^{4}$. Right: Combined results for $R\left(D^{(*)}\right)$ and $b \rightarrow s \ell^{+} \ell^{-}$, and contours for $\left[\operatorname{Br}\left(B \rightarrow K \tau^{+} \mu^{-}\right)+\operatorname{Br}\left(B \rightarrow K \tau^{-} \mu^{+}\right)\right] / 2$. The red region is preferred by the global fit to $b \rightarrow s \ell^{+} \ell^{-}$data.

[5] F. Betti [LHCb Collaboration], arXiv:1705.10651 [hep-ex].

[6] Y. Amhis et al., arXiv:1612.07233 [hep-ex].

[7] Fontana, Marianna [LHCb Collaboration], LHCb Status Report, talk given at CERN on the 13.09.2017: https://indico.cern.ch/event/658856/contributions/ 2686351/attachments/1522412/2379024/talk_LHCb_MFontana.pdf

[8] R. Aaij et al. [LHCb Collaboration], Phys. Rev. Lett. 113, 151601 (2014) doi:10.1103/PhysRevLett.113.151601 [arXiv:1406.6482 [hep-ex]].

[9] R. Aaij et al. [LHCb Collaboration], JHEP 1708, 055 (2017) doi:10.1007/JHEP08(2017)055 [arXiv:1705.05802 [hep-ex]].

[10] W. Altmannshofer, P. Stangl and D. M. Straub, Phys. Rev. D 96, no. 5, 055008 (2017) doi:10.1103/PhysRevD.96.055008 [arXiv:1704.05435 [hep-ph]].

[11] G. D'Amico, M. Nardecchia, P. Panci, F. Sannino, A. Strumia, R. Torre and A. Urbano, JHEP 1709, 010 (2017) doi:10.1007/JHEP09(2017)010 [arXiv:1704.05438 [hep-ph]].

[12] G. Hiller and I. Nisandzic, Phys. Rev. D 96, no. 3, 035003 (2017) doi:10.1103/PhysRevD.96.035003 [arXiv:1704.05444 [hep-ph]].

[13] L. S. Geng, B. Grinstein, S. Jäger, J. Martin Camalich, X. L. Ren and R. X. Shi, arXiv:1704.05446 [hep-ph].

[14] T. Hurth, F. Mahmoudi, D. Martinez Santos and S. Neshatpour, arXiv:1705.06274 [hep-ph].

[15] R. Aaij et al. [LHCb Collaboration], JHEP 1602, 104 (2016) doi:10.1007/JHEP02(2016)104 [arXiv:1512.04442 [hep-ex]].

[16] B. Capdevila, A. Crivellin, S. Descotes-Genon, J. Matias and J. Virto, arXiv:1704.05340 [hep-ph].

[17] G. W. Bennett et al. [Muon g-2 Collaboration], Phys. Rev. D 73, 072003 (2006) doi:10.1103/PhysRevD.73.072003 [hep-ex/0602035]. 
[18] A. Nyffeler, Phys. Rev. D 94, no. 5, 053006 (2016) doi:10.1103/PhysRevD.94.053006 [arXiv:1602.03398 [hep-ph]].

[19] A. Lusiani, Nucl. Part. Phys. Proc. 287-288, 29 (2017). doi:10.1016/j.nuclphysbps.2017.03.038

[20] A. J. Buras, M. Gorbahn, S. Jäger and M. Jamin, JHEP 1511, 202 (2015) doi:10.1007/JHEP11(2015)202 [arXiv:1507.06345 [hep-ph]].

[21] J. P. Lees et al. [BaBar Collaboration], Phys. Rev. D 85, 031102 (2012) Erratum: [Phys. Rev. D 85, 099904 (2012)] doi:10.1103/PhysRevD.85.099904, 10.1103/PhysRevD.85.031102 [arXiv:1109.1527 [hep-ex]].

[22] A. Crivellin, C. Greub and A. Kokulu, Phys. Rev. D 86, 054014 (2012) doi:10.1103/PhysRevD.86.054014 [arXiv:1206.2634 [hep-ph]].

[23] M. Tanaka and R. Watanabe, Phys. Rev. D 87, no. 3, 034028 (2013) doi:10.1103/PhysRevD.87.034028 [arXiv:1212.1878 [hep-ph]].

[24] A. Celis, M. Jung, X. Q. Li and A. Pich, JHEP 1301, 054 (2013) doi:10.1007/JHEP01(2013)054 [arXiv:1210.8443 [hep-ph]].

[25] A. Crivellin, A. Kokulu and C. Greub, Phys. Rev. D 87, no. 9, 094031 (2013) doi:10.1103/PhysRevD.87.094031 [arXiv:1303.5877 [hep-ph]].

[26] A. Crivellin, J. Heeck and P. Stoffer, Phys. Rev. Lett. 116, no. 8, 081801 (2016) doi:10.1103/PhysRevLett.116.081801 [arXiv:1507.07567 [hep-ph]].

[27] R. Alonso, B. Grinstein and J. Martin Camalich, JHEP 1510, 184 (2015) doi:10.1007/JHEP10(2015)184 [arXiv:1505.05164 [hep-ph]].

[28] L. Calibbi, A. Crivellin and T. Ota, Phys. Rev. Lett. 115, 181801 (2015) doi:10.1103/PhysRevLett.115.181801 [arXiv:1506.02661 [hep-ph]].

[29] M. Bauer and M. Neubert, Phys. Rev. Lett. 116, no. 14, 141802 (2016) doi:10.1103/PhysRevLett.116.141802 [arXiv:1511.01900 [hep-ph]].

[30] D. Becirevic, S. Fajfer, N. Kosnik and O. Sumensari, Phys. Rev. D 94, no. 11, 115021 (2016) doi:10.1103/PhysRevD.94.115021 [arXiv:1608.08501 [hep-ph]].

[31] R. Barbieri, C. W. Murphy and F. Senia, Eur. Phys. J. C 77, no. 1, 8 (2017) doi:10.1140/epjc/s10052-016-4578-7 [arXiv:1611.04930 [hep-ph]].

[32] A. Greljo, G. Isidori and D. Marzocca, JHEP 1507, 142 (2015) doi:10.1007/JHEP07(2015)142 [arXiv:1506.01705 [hep-ph]].

[33] A. G. Akeroyd and C. H. Chen, arXiv:1708.04072 [hep-ph].

[34] M. Freytsis, Z. Ligeti and J. T. Ruderman, Phys. Rev. D 92, no. 5, 054018 (2015) doi:10.1103/PhysRevD.92.054018 [arXiv:1506.08896 [hep-ph]].

[35] D. A. Faroughy, A. Greljo and J. F. Kamenik, Phys. Lett. B 764, 126 (2017) doi:10.1016/j.physletb.2016.11.011 [arXiv:1609.07138 [hep-ph]].

[36] F. Feruglio, P. Paradisi and A. Pattori, Phys. Rev. Lett. 118, no. 1, 011801 (2017) doi:10.1103/PhysRevLett.118.011801 [arXiv:1606.00524 [hep-ph]].

[37] A. Crivellin, D. Müller and T. Ota, JHEP 1709, 040 (2017) doi:10.1007/JHEP09(2017)040 [arXiv:1703.09226 [hep-ph]].

[38] L. Di Luzio, A. Greljo and M. Nardecchia, arXiv:1708.08450 [hep-ph]. 
[39] D. Buttazzo, A. Greljo, G. Isidori and D. Marzocca, arXiv:1706.07808 [hep-ph].

[40] L. Calibbi, A. Crivellin and T. Li, arXiv:1709.00692 [hep-ph].

[41] X. Q. Li, Y. D. Yang and X. Zhang, JHEP 1608, 054 (2016) doi:10.1007/JHEP08(2016)054 [arXiv:1605.09308 [hep-ph]].

[42] W. Altmannshofer, P. S. B. Dev and A. Soni, arXiv:1704.06659 [hep-ph].

[43] S. Descotes-Genon, J. Matias and J. Virto, Phys. Rev. D 88, 074002 (2013) doi:10.1103/PhysRevD.88.074002 [arXiv:1307.5683 [hep-ph]].

[44] R. Gauld, F. Goertz and U. Haisch, Phys. Rev. D 89, 015005 (2014) doi:10.1103/PhysRevD.89.015005 [arXiv:1308.1959 [hep-ph]].

[45] A. J. Buras and J. Girrbach, JHEP 1312, 009 (2013) doi:10.1007/JHEP12(2013)009 [arXiv:1309.2466 [hep-ph]].

[46] W. Altmannshofer, S. Gori, M. Pospelov and I. Yavin, Phys. Rev. D 89, 095033 (2014) doi:10.1103/PhysRevD.89.095033 [arXiv:1403.1269 [hep-ph]].

[47] A. Crivellin, G. D’Ambrosio and J. Heeck, Phys. Rev. Lett. 114, 151801 (2015) doi:10.1103/PhysRevLett.114.151801 [arXiv:1501.00993 [hep-ph]].

[48] A. Crivellin, G. D’ Ambrosio and J. Heeck, Phys. Rev. D 91, no. 7, 075006 (2015) doi:10.1103/PhysRevD.91.075006 [arXiv:1503.03477 [hep-ph]].

[49] A. Crivellin, L. Hofer, J. Matias, U. Nierste, S. Pokorski and J. Rosiek, Phys. Rev. D 92, no. 5, 054013 (2015) doi:10.1103/PhysRevD.92.054013 [arXiv:1504.07928 [hep-ph]].

[50] A. Crivellin, J. Fuentes-Martin, A. Greljo and G. Isidori, Phys. Lett. B 766, 77 (2017) doi:10.1016/j.physletb.2016.12.057 [arXiv:1611.02703 [hep-ph]].

[51] B. Gripaios, M. Nardecchia and S. A. Renner, JHEP 1505, 006 (2015) doi:10.1007/JHEP05(2015)006 [arXiv:1412.1791 [hep-ph]].

[52] S. Fajfer and N. Kosnik, Phys. Lett. B 755, 270 (2016) doi:10.1016/j.physletb.2016.02.018 [arXiv:1511.06024 [hep-ph]].

[53] B. Gripaios, M. Nardecchia and S. A. Renner, JHEP 1606, 083 (2016) doi:10.1007/JHEP06(2016)083 [arXiv:1509.05020 [hep-ph]].

[54] P. Arnan, L. Hofer, F. Mescia and A. Crivellin, JHEP 1704, 043 (2017) doi:10.1007/JHEP04(2017)043 [arXiv:1608.07832 [hep-ph]].

[55] D. Becirevic and O. Sumensari, JHEP 1708, 104 (2017) doi:10.1007/JHEP08(2017)104 [arXiv:1704.05835 [hep-ph]].

[56] J. F. Kamenik, Y. Soreq and J. Zupan, arXiv:1704.06005 [hep-ph].

[57] W. Altmannshofer and D. M. Straub, Eur. Phys. J. C 73, 2646 (2013) doi:10.1140/epjc/s10052-013-2646-9 [arXiv:1308.1501 [hep-ph]].

[58] F. Mahmoudi, S. Neshatpour and J. Virto, Eur. Phys. J. C 74, no. 6, 2927 (2014) doi:10.1140/epjc/s10052-014-2927-y [arXiv:1401.2145 [hep-ph]].

[59] D. Stockinger, J. Phys. G 34, R45 (2007) doi:10.1088/0954-3899/34/2/R01 [hep-ph/0609168].

[60] A. Crivellin, J. Girrbach and U. Nierste, Phys. Rev. D 83, 055009 (2011) doi:10.1103/PhysRevD.83.055009 [arXiv:1010.4485 [hep-ph]]. 
[61] A. Djouadi, T. Kohler, M. Spira and J. Tutas, Z. Phys. C 46, 679 (1990). doi:10.1007/BF01560270

[62] W. Buchmuller, R. Ruckl and D. Wyler, Phys. Lett. B 191, 442 (1987) Erratum: [Phys. Lett. B 448, 320 (1999)]. doi:10.1016/S0370-2693(99)00014-3, 10.1016/0370-2693(87)90637-X

[63] E. Coluccio Leskow, G. D’Ambrosio, A. Crivellin and D. Müller, Phys. Rev. D 95, no. 5, 055018 (2017) doi:10.1103/PhysRevD.95.055018 [arXiv:1612.06858 [hep-ph]].

[64] H. Baer et al., arXiv:1306.6352 [hep-ph].

[65] A. J. Buras, D. Buttazzo and R. Knegjens, JHEP 1511, 166 (2015) doi:10.1007/JHEP11(2015)166 [arXiv:1507.08672 [hep-ph]].

[66] A. J. Buras and F. De Fazio, JHEP 1603, 010 (2016) doi:10.1007/JHEP03(2016)010 [arXiv:1512.02869 [hep-ph]].

[67] M. Endo, T. Kitahara, S. Mishima and K. Yamamoto, Phys. Lett. B 771, 37 (2017) doi:10.1016/j.physletb.2017.05.026 [arXiv:1612.08839 [hep-ph]].

[68] V. Cirigliano, W. Dekens, J. de Vries and E. Mereghetti, Phys. Lett. B 767, 1 (2017) doi:10.1016/j.physletb.2017.01.037 [arXiv:1612.03914 [hep-ph]].

[69] T. Kitahara, U. Nierste and P. Tremper, Phys. Rev. Lett. 117, no. 9, 091802 (2016) doi:10.1103/PhysRevLett.117.091802 [arXiv:1604.07400 [hep-ph]].

[70] M. Endo, S. Mishima, D. Ueda and K. Yamamoto, Phys. Lett. B 762, 493 (2016) doi:10.1016/j.physletb.2016.10.009 [arXiv:1608.01444 [hep-ph]].

[71] A. Crivellin, G. D’Ambrosio, T. Kitahara and U. Nierste, Phys. Rev. D 96, no. 1, 015023 (2017) doi:10.1103/PhysRevD.96.015023 [arXiv:1703.05786 [hep-ph]].

[72] A. Crivellin and M. Davidkov, Phys. Rev. D 81, 095004 (2010) doi:10.1103/PhysRevD.81.095004 [arXiv:1002.2653 [hep-ph]].

[73] J. A. Bailey et al. [SWME Collaboration], Phys. Rev. D 92, no. 3, 034510 (2015) doi:10.1103/PhysRevD.92.034510 [arXiv:1503.05388 [hep-lat]].

[74] The ATLAS collaboration [ATLAS Collaboration], ATLAS-CONF-2016-078.

[75] CMS Collaboration [CMS Collaboration], CMS-PAS-SUS-16-015.

[76] G. Aad et al. [ATLAS Collaboration], JHEP 1510, 054 (2015) doi:10.1007/JHEP10(2015)054 [arXiv:1507.05525 [hep-ex]].

[77] D. Becirevic, N. Kosnik, O. Sumensari and R. Zukanovich Funchal, JHEP 1611, 035 (2016) doi:10.1007/JHEP11(2016)035 [arXiv:1608.07583 [hep-ph]].

[78] Y. Cai, J. Gargalionis, M. A. Schmidt and R. R. Volkas, arXiv:1704.05849 [hep-ph].

[79] J. P. Lees et al. [BaBar Collaboration], Phys. Rev. D 86, 012004 (2012) doi:10.1103/PhysRevD.86.012004 [arXiv:1204.2852 [hep-ex]].

[80] P. Q. Hung, A. J. Buras and J. D. Bjorken, Phys. Rev. D 25, 805 (1982). doi:10.1103/PhysRevD.25.805

[81] A. Crivellin, D. Mueller, A. Signer and Y. Ulrich, arXiv:1706.08511 [hep-ph]. 